\title{
Smart phone ophthalmoscopy: a potential replacement for the direct ophthalmoscope
}

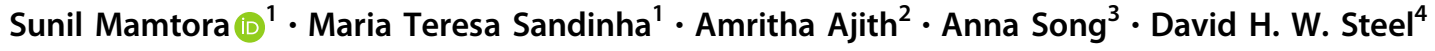

Received: 27 December 2017 / Revised: 29 May 2018 / Accepted: 18 June 2018 / Published online: 24 July 2018

(c) The Royal College of Ophthalmologists 2018

\begin{abstract}
Purpose The purpose of this study is to evaluate a commercially available smartphone ophthalmoscope, D-EYE, as compared with the direct ophthalmoscope when used by a cohort of final-year medical students in a prospective study.

Methods Two-hundred fundal examinations were performed on the eyes of 10 mannequins featuring 5 unique fundal images by 20 final-year medical students from Newcastle University. Each student examined the five fundal images twice, once each with a direct ophthalmoscope and D-EYE in a random order. Students recorded their findings at the optic nerve, macula, and retina in an objective questionnaire, and the findings were analysed by an observer masked to the examination technique.

Results Students provided more accurate clinical descriptions of their findings when using D-EYE as opposed to using the direct ophthalmoscope $(p<0.05)$. In addition, we found that students were overall more likely to make a correct diagnosis based on their findings when using D- EYE compared with the direct ophthalmoscope.

Conclusion Our study suggests that the use of a smartphone-based alternative to the direct ophthalmoscope may improve the accuracy and quality of fundal examinations by non-ophthalmologists.
\end{abstract}

\section{Introduction}

Direct ophthalmoscopy (DO) poses a number of clinical challenges. Although the high magnification afforded by its optical system may provide a good image of the optic disc, the field of view is considerably narrow at approximately two disc diameters. This view is even smaller in myopic patients, a cohort whose prevalence is rapidly increasing [1]. A complete examination of the retina, which would comprise of more than a hundred fields of view, is therefore not feasible.

Mydriasis aids DO considerably but is commonly avoided in the acute medical setting, as this prevents examination of the pupillary reflexes. Pupillary dilating agents are

Sunil Mamtora

s.p.mamtora@hotmail.com

Sunderland Eye Infirmary, Sunderland, UK

2 Sunderland Royal Hospital, Sunderland, UK

3 Newcastle University, Newcastle upon Tyne, UK

4 Institute of Genetic Medicine, Newcastle University, Newcastle upon Tyne, UK also avoided due to a fear of inducing angle closure glaucoma [2]. This is despite the risk being proven to be vanishingly small [3].

Given these factors, it is therefore not surprising that medical students lack confidence in using the direct ophthalmoscope [4]. Time spent in Ophthalmology at medical school has decreased significantly and proficiency amongst medical students at DO, which is rarely tested in undergraduate examinations, has also declined $[5,6]$. The question as to whether or not undergraduate medical students should be taught DO has been the source of much controversy $[7,8]$.

Foundation doctors also report limited confidence in DO with one study finding that less than one in five felt confident at identifying papilloedema [9]. This is despite DO being listed as a fundamental competency in the Foundation Programme curriculum [10].

Numerous innovative devices are either currently or soon to be available, which allow for digital fundus imaging using mobile devices. These devices generally consist of a small lens adapter, which attaches to the back of a mobile phone camera and have been found to generate high-quality images. D-EYE, one of the currently available devices, was selected for use in our study and is shown in Fig. 1 (f). 


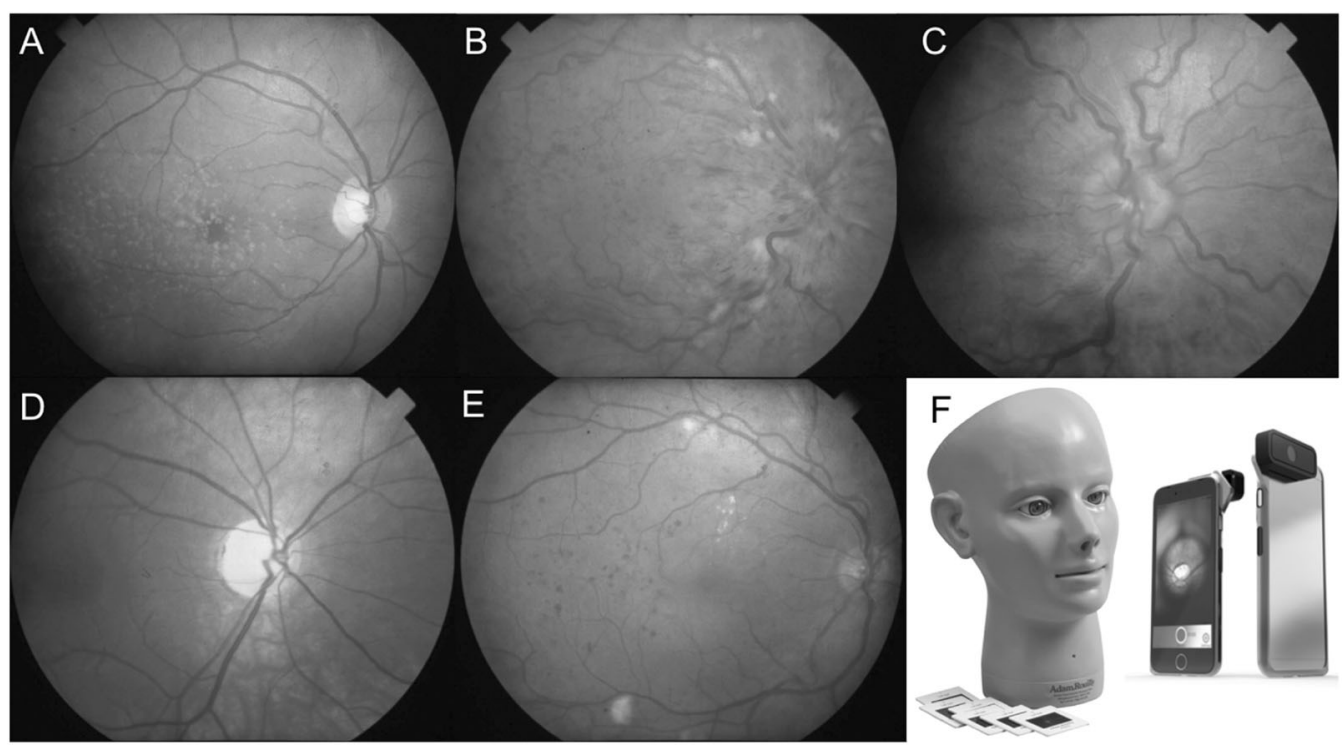

Fig. 1 a-e Film slides as used in the study. f Adam Rouilly AR303 head mannequin and D-EYE attached to an Apple iPhone

Despite the increasing use of smartphone-based fundus cameras such as D-EYE, there has been little study to formally assess the clinical utility of such devices in assisting the diagnosis of fundoscopically identifiable diseases particularly by novice clinicians. We therefore designed a prospective study to evaluate the diagnostic validity of DEYE as compared with the direct ophthalmoscope when used by a cohort of final-year medical students.

\section{Methods}

Two-hundred fundal examinations were performed on the eyes of mannequins by 20 final-year medical students from Newcastle University, UK. The study took place at Sunderland Royal Hospital, Sunderland, UK, and formed a part of final-year medical student Ophthalmology teaching.

Students independently examined the eyes of 10 mannequins featuring 5 unique fundal images. Examinations were performed with a direct ophthalmoscope and D-EYE, a commercially available system which allows users to examine the fundus using an Apple iPhone device. Each student therefore examined the five unique fundal images twice, once each with a direct ophthalmoscope $(3.5 \mathrm{~V}$ Standard Ophthalmoscope, Welch Allyn, USA) and DEYE.

The D-EYE system generates images using co-axial illumination and a beam splitter. In our study D-EYE was used alongside Apple iPhone 5S devices, which captured images with a resolution of $3264 \times 2448$ pixels. The D-EYE system was used through a mobile application on the smartphone, allowing the user to capture both photos and videos that could be stored and played back as necessary.

Head mannequins (Adam Rouilly AR303, UK) with model eyes with a $3 \mathrm{~mm}$ fixed pupil diameter and ocular fundus created using film slides inserted behind the globe (Fig. 1) were used for fundoscopy to standardise the experimental conditions.

The five unique fundal images that were installed into the mannequins were chosen as being representative of common and important fundoscopically identifiable eye conditions. The slides chosen featured a mixture of pathology affecting the optic nerve head, macula and the more peripheral retina. These slides showed optic atrophy, dry agerelated macular degeneration, central retinal vein occlusion, papilloedema, and pre-proliferative diabetic retinopathy.

The students were randomly allocated into 2 groups each containing 10 students. The order of the mannequins, as well as whether the mannequin was to be examined by the direct ophthalmoscope or D-EYE was also randomised. These parameters were maintained across both groups.

All students received a tutorial, supervised by a consultant Ophthalmologist, on how to use both the direct Ophthalmoscope and D-EYE prior to the session which was performed under examination conditions. Furthermore, students received a comprehensive demonstrating a wide range of fundoscopically identifiable conditions including all those featured in our study.

All students had received regular teaching on the use of a direct ophthalmoscope during their previous years at medical school and were experienced in its use. None of the medical students had previously used D-EYE. 
Fig. 2 Questionnaire used by students to record their findings using either the direct ophthalmoscope or D-EYE

\begin{tabular}{|c|c|c|c|c|c|}
\hline \multicolumn{6}{|c|}{ Examination findings: } \\
\hline Eye: & Left & Right & \multicolumn{3}{|l|}{ Unsure } \\
\hline Optic disc & Normal & Swollen & Pale & Unsure & Not visualised \\
\hline Macula: & Normal & Haemorrhage & Drusen & Unsure & Not visualised \\
\hline Retina: & Normal & Haemorrhage & Exudate & Unsure & Not visualised \\
\hline \multirow[t]{4}{*}{ Diagnosis } & \multicolumn{3}{|c|}{ Normal Retina } & \multicolumn{2}{|c|}{ Age related macular degeneration } \\
\hline & \multicolumn{2}{|c|}{ Central retinal vein occlusion } & & \multicolumn{2}{|c|}{ Hypertensive retinopathy } \\
\hline & \multicolumn{2}{|c|}{ Papilloedema } & & \multicolumn{2}{|l|}{ Disc cupping } \\
\hline & \multicolumn{2}{|c|}{ Optic atrophy } & & \multicolumn{2}{|c|}{ Diabetic retinopathy } \\
\hline \multicolumn{2}{|c|}{ Certainty of diagnosis } & $0 \%$ & $<50 \%$ & $>50 \%$ & $100 \%$ \\
\hline
\end{tabular}

The only support provided during the session was of a technical nature, e.g., if a direct ophthalmoscope had no batteries or if a student was unable to access the app required to use D-EYE. Each student spent 3 min with each mannequin before rotating onto the next mannequin in order.

An objective questionnaire was designed to allow comparison of the quality of examination findings between the two different examination methods (Fig. 2). Students were asked to document their findings regarding the optic nerve, macula, and retina. The questionnaire went through an extensive validation process, which included review by consultant ophthalmologists, nursing staff who provided feedback about the language and wording of the questionnaire, and medical students who trialled the questionnaire before the study.

One point was awarded for each correct clinical description of each anatomical area with a maximum of three points per examination. As each slide was examined by 20 students across 2 separate groups, the total available score for each slide was 60 points.

Students were then asked to make a diagnosis based on their findings and state the level of certainty with which they made their diagnosis.

Following the session all participants were asked whether they felt that the use of a smartphone-based alternative to the direct ophthalmoscope could improve clinical examination by non-Ophthalmologists on a Lickert scale.

Testing for normality was performed on the complete data set and data sets from individual groups using the Shapiro-Wilk test. The overall data set was found to be normally distributed and statistical analysis was performed using the two-tailed $t$-test. Our sub-group data sets were not found to be normally distributed and statistical analysis of sub-groups was performed using the Wilcoxon signed-rank test. A $p$-value of $<0.05$ was considered to be significant.

Newcastle University granted ethical approval for this study and all students gave their consent for their participation.

\section{Results}

We found that students provided more accurate clinical descriptions of their findings when using D-EYE as opposed to using the direct ophthalmoscope. This finding was consistent across both groups and was statistically significant $(p<0.05)$. The total score for students in both groups using D-EYE was 199 (66\%) and 138 (46\%) with the direct ophthalmoscope (Tables 1, 2, and 3).

\section{Discussion}

The students' performance was better overall when D-EYE was used to examine the fundi of mannequins compared with when the direct ophthalmoscope was used. Our results showed several statistically significant and clinically relevant differences, although there were some discrepancies in our findings.

Students were able to provide more accurate clinical descriptions of the fundal images in all slides, except for papilloedema. Here the results suggested an equivalent performance by the direct ophthalmoscope and the D-EYE with the sign being equally difficult to discern (48\% vs. $47 \%$ in terms of points).

Similarly, there was no difference in the ability of students being able to identify the presence of a swollen optic 
Table 1 Table showing total number of points scored for accurately describing clinical findings by students in both groups using either D-EYE or the direct ophthalmoscope in each of the five clinical conditions

\begin{tabular}{llll}
\hline Pathology & $\begin{array}{l}\text { D-EYE (maximum } \\
\text { score out of 60) }\end{array}$ & $\begin{array}{l}\text { Direct ophthalmoscope } \\
\text { (maximum score out of 60) }\end{array}$ & $P$-value \\
\hline $\begin{array}{l}\text { Dry age-related macular degeneration } \\
\text { (AMD) }\end{array}$ & $47(78 \%)$ & $28(47 \%)$ & $P<0.05$ \\
Central retinal vein occlusion (CRVO) & $31(52 \%)$ & $15(25 \%)$ & $P<0.05$ \\
Papilloedema & $28(47 \%)$ & $29(48 \%)$ & $P=0.52$ \\
Optic atrophy & $50(83 \%)$ & $34(57 \%)$ & $P=0.08$ \\
Pre-proliferative diabetic retinopathy & $43(72 \%)$ & $32(53 \%)$ & $p=<0.05$ \\
\hline
\end{tabular}

Table 2 Table showing students' ability to correctly describe their findings by different anatomical location for each fundus slide

\begin{tabular}{llll}
\hline Diagnosis & $\begin{array}{l}\text { Anatomical } \\
\text { location }\end{array}$ & $\begin{array}{l}\text { D-EYE (maximum } \\
\text { score out of 20) }\end{array}$ & $\begin{array}{l}\text { Direct ophthalmoscope } \\
\text { (maximum score out of 20) }\end{array}$ \\
\hline $\begin{array}{l}\text { Dry age-related macular } \\
\text { degeneration (AMD) }\end{array}$ & Optic disc & $17(85 \%)$ & $9(45 \%)$ \\
& Macula & $17(85 \%)$ & $7(35 \%)$ \\
& Retina & $13(65 \%)$ & $12(60 \%)$ \\
Central retinal vein & Optic disc & $13(65 \%)$ & $3(15 \%)$ \\
occlusion (CRVO) & Macula & $6(30 \%)$ & $1(5 \%)$ \\
& Retina & $12(60 \%)$ & $11(55 \%)$ \\
Papilloedema & Optic disc & $12(60 \%)$ & $10(50 \%)$ \\
& Macula & $8(40 \%)$ & $8(40 \%)$ \\
& Retina & $8(40 \%)$ & $11(55 \%)$ \\
Optic atrophy & Optic disc & $16(80 \%)$ & $12(60 \%)$ \\
& Macula & $17(85 \%)$ & $11(55 \%)$ \\
& Retina & $17(85 \%)$ & $12(55 \%)$ \\
Pre-proliferative diabetic & Optic disc & $18(90 \%)$ & $6(30 \%)$ \\
retinopathy & Macula & $6(30 \%)$ & $14(70 \%)$ \\
& Retina & $19(95 \%)$ & \\
\hline
\end{tabular}

\begin{tabular}{|c|c|c|c|}
\hline Pathology & $\begin{array}{l}\text { D-EYE } \\
\text { (maximum score 20) }\end{array}$ & $\begin{array}{l}\text { Direct ophthalmoscope } \\
\text { (maximum score 20) }\end{array}$ & $P$-value \\
\hline $\begin{array}{l}\text { Dry age-related macular } \\
\text { degeneration (AMD) }\end{array}$ & $15(75 \%)$ & $6(30 \%)$ & $P<0.05$ \\
\hline $\begin{array}{l}\text { Central retinal vein occlusion } \\
(\mathrm{CRVO})\end{array}$ & $8(40 \%)$ & $6(30 \%)$ & $P=0.48$ \\
\hline Papilloedema & $6(30 \%)$ & $6(30 \%)$ & $P=0.78$ \\
\hline Optic atrophy & $4(20 \%)$ & $4(20 \%)$ & $P=0.66$ \\
\hline $\begin{array}{l}\text { Pre-proliferative diabetic } \\
\text { retinopathy }\end{array}$ & $14(70 \%)$ & $8(40 \%)$ & $P=0.10$ \\
\hline
\end{tabular}

Table 3 Table showing the number and percentage of students in each group, who were able to make a correct diagnosis in each fundal image disc $60 \%$ D-EYE vs. $50 \%$ DO $(p=0.26)$. We postulate that as the direct ophthalmoscope is able to provide a good quality image of the centrally located optic disc with a highly magnified view the D-EYE does not offer any advantages for this diagnosis. Although optic disc swelling was identified significantly more frequently in CRVO, we suggest this may have been due to students being able to readily make a correct diagnosis after visualising haemorrhages throughout the retina and thereafter assuming the disc to be swollen.

Students made a correct diagnosis using D-EYE significantly more frequently in dry AMD and pre-proliferative 
diabetic retinopathy. Our study found that the ability for students to correctly diagnose papilloedema and optic atrophy between D-EYE and the direct ophthalmoscope was equivalent. The authors suggest that the reason that students were able to less frequently make a correct diagnosis of papilloedema could have been due to the presence of hypertensive retinopathy and central retinal vein occlusion, which featured as alternative options in the questionnaire and are also associated with optic disc swelling.

The slide demonstrating optic atrophy only showed pallor of the optic disc with no other positive findings. Students commonly diagnosed this slide as demonstrating no pathology. Although students infrequently made a correct diagnosis, in this case they were able to describe their findings accurately as $80 \%$ of students using D-EYE and $60 \%$ using the direct ophthalmoscope identified the disc as being pale. We suspect that students were less likely to make a correct diagnosis in this condition despite providing accurate clinical descriptions as optic atrophy is not a frequently taught condition in the undergraduate ophthalmology curriculum.

There were a number of limitations to our study. The performance of medical students in our study may not necessarily be representative of practicing healthcare professionals who may not have had practice or training in the use of the direct ophthalmoscope for a considerable amount of time. Furthermore, students received a tutorial immediately before the study outlining common fundal abnormalities and how to use the direct ophthalmoscope. All conditions featured in our study were demonstrated in this tutorial. This may artificially lessen the gap between D-EYE and the direct ophthalmoscope. Large studies have found that even experienced clinicians often perform DO poorly [11].

Due to the nature of the study, participants could not be blinded to the device they were using to record their findings. In addition, our study used mannequins, in order to produce reproducible and objective clinical findings. The mannequins that we used could be argued to be not representative of examining a patient in the clinical setting.

A further major limitation of our study was that students examined the same film slides twice using each imaging modality, which could influence the findings when examining the same slide for the second time. To minimise this, students visited each station in a randomised order.

Students were asked about their opinions relating to the potential for D-EYE to be used in clinical medicine. Although the vast majority (95\%) felt that D-EYE could improve quality of care, specific comments raised concerns relating to the availability of smartphones in hospitals for this purpose and how securely data would be stored once captured.
Tablets and smartphones are becoming used increasingly within hospitals for a variety of purposes including electronic documentation, monitoring of vital signs, and even as a method of transmitting secure messages between healthcare professionals [12]. The use of such readily available devices to perform high-quality fundal examination may allay concerns regarding doctors using their personal mobile devices to capture confidential patient images. The sitting government has already pledged to set aside more than $£ 4 \mathrm{bn}$ to improve the use of technology in the National Health Service (NHS) in England and has plans to introduce NHSapproved apps that are able to link directly into medical records [13]. The ability to perform smartphone-based ophthalmoscopy could potentially allow for fundus images to be stored to the medical record for future comparison. Clinicians could use the digitally stored images to obtain a specialist opinion without arranging for a clinic visit.

Drawbacks to mobile fundus photography are as with any mobile based technology used in healthcare, namely secure transfer of patient data and a source of power. The field of view provided by mobile based fundus cameras in the undilated pupil is already improved compared to the direct ophthalmoscope but could be increased further in the future through the use of software to intelligently stitch multiple images together, which have been captured from different regions of the peripheral retina [14].

Other studies have evaluated the potential of smartphone-based alternatives to the direct ophthalmoscope. One study found that PEEK, a similar smartphonebased fundus camera, was able to capture high-quality images of the optic disc whose grading's were comparable to images taken by a conventional desktop retinal camera [15]. Other studies using D-EYE, the smartphone-based fundus camera system that was used in our study, have found that clinicians using D-EYE were able to reliably grade cup-disc ratios in patients with glaucoma and identify features of hypertensive retinopathy [16, 17]. Our study differs from the existing literature in that it evaluated the ability of soon to be clinicians in their use of a smartphone ophthalmoscope compared with the traditional direct ophthalmoscope. We therefore feel that our study uniquely demonstrates the potential of smartphone ophthalmoscopy in clinical medicine.

\section{Conclusion}

In support of the existing literature, our study suggests that the use of a smartphone alternative to the direct ophthalmoscope can improve the accuracy and quality of fundal examinations by non-ophthalmologists. Ninety-five percent of the study participants shared this view. 
The use of smartphone-based technology in medicine and healthcare is rapidly evolving. The authors of this study feel that it may not be long before smartphone fundal imaging technologies are able to replace the direct ophthalmoscope in clinical medicine.

\section{Summary}

\section{What was known before:}

- Experienced clinicians often miss important findings when performing direct ophthalmoscopy and direct ophthalmoscopy itself is critically underperformed in the general medical setting.

- Smartphone ophthalmoscopy has been shown to be able to capture high-quality images of the optic disc and grading using these images was comparable to a conventional desktop retinal camera.

\section{What this study adds:}

- Smartphone ophthalmoscopy improves the ability to correctly identify fundal features and the diagnostic accuracy when used by final-year medical students.

\section{Compliance with ethical standards}

Conflict of interest The authors declare that they have no conflict of interest.

\section{References}

1. Williams KM, Bertelsen G, Cumberland P, Wolfram C, Verhoeven VJ, Anastasopoulos E, et al. Increasing prevalence of myopia in Europe and the impact of education. Ophthalmology. 2015;122:1489-97.

2. Ah-kee EY, Egong E, Shafi A, Lim LT, Yim JL. A review of drug-induced acute angle closure glaucoma for nonophthalmologists. Qatar Med J. 2015;2015:6.
3. Pandit RJ, Taylor R. Mydriasis and glaucoma: exploding the myth. A systematic review. Diabet Med. 2000;17:693-9.

4. Gupta RR, Lam WC. Medical students' self-confidence in performing direct ophthalmoscopy in clinical training. Can J Ophthalmol. 2006;41:169-74.

5. Baylis O, Murray PI, Dayan M. Undergraduate ophthalmology education-a survey of UK medical schools. Med Teach. 2011;33:468-71.

6. Lippa LM, Boker J, Duke A, Amin A. A novel 3-year longitudinal pilot study of medical students' acquisition and retention of screening eye examination skills. Ophthalmology. 2006; 113:133-9.

7. Yusuf IH, Salmon JF, Patel CK. Direct ophthalmoscopy should be taught to undergraduate medical students-yes. Eye (Lond). 2015;29:987-9.

8. Purbrick RM, Chong NV. Direct ophthalmoscopy should be taught to undergraduate medical students--no. Eye (Lond). 2015;29:990-1.

9. Nicholl DJ, Yap CP, Cahill V, Appleton J, Willetts E, Sturman S. The TOS study: can we use our patients to help improve clinical assessment? J R Coll Physicians Edinb. 2012;42:306-10.

10. Colleges AoMR. The UK Foundation Programme Curriculum. 2012. http://www.foundationprogramme.nhs.uk/curriculum/.

11. Lamirel C, Bruce BB, Wright DW, Delaney KP, Newman NJ, Biousse V. Quality of nonmydriatic digital fundus photography obtained by nurse practitioners in the emergency department: the FOTO-ED study. Ophthalmology. 2012; 119:617-24.

12. Alexander SM, Nerminathan A, Harrison A, Phelps M, Scott KM. Prejudices and perceptions: patient acceptance of mobile technology use in health care. Intern Med J. 2015;45:1179-81.

13. MP DoHaTRHJH. New plans to expand the use of digital technology across the NHS. 2016. https://www.gov.uk/government/ news/new-plans-to-expand-the-use-of-digital-technology-acrossthe-nhs.

14. Bolster NM, Giardini ME, Livingstone IAT, Bastawrous A. How the smartphone is driving the eye-health imaging revolution. Expert Rev Ophthalmol. 2014;9:475-85.

15. Bastawrous A, Giardini M, Bolster NM, et al. CLinical validation of a smartphone-based adapter for optic disc imaging in kenya. JAMA Ophthalmol. 2016;134:151-8.

16. Muiesan ML, Salvetti M, Paini A, Riviera M, Pintossi $\mathrm{C}$, Bertacchini $\mathrm{F}$, et al. Ocular fundus photography with a smartphone device in acute hypertension. J Hypertens. 2017;35:1660-5.

17. Russo A, Mapham W, Turano R, Costagliola C, Morescalchi F, Scaroni N, et al. Comparison of smartphone ophthalmoscopy with slit-lamp biomicroscopy for grading vertical cup-to-disc ratio. J Glaucoma. 2016;25:e777-81. 